\title{
Distributed roughness effects on transitional and turbulent boundary layers
}

\section{Distributed roughness effects on transitional and turbulent boundary layers}

\author{
Nagabhushana Rao Vadlamani ${ }^{1}$ - Paul \\ G. Tucker ${ }^{1}$ - Paul Durbin ${ }^{2}$
}

Received: date / Accepted: date

\begin{abstract}
A numerical investigation is carried out to study the transition of a subsonic boundary layer on a flat plate with roughness elements distributed over the entire surface. Post-transition, the effect of surface roughness on a spatially developing turbulent boundary layer (TBL) is explored. In the transitional regime, the onset of flow transition predicted by the current simulations is in agreement with the experimentally based correlations proposed in the literature. Transition mechanisms are shown to change significantly with the increasing roughness height. Roughness elements that are inside the boundary layer create an elevated shear layer and alternating high and low speed streaks near the wall. Secondary sinuous instabilities on the streaks destabilize the shear layer promoting transition to turbulence. For the roughness topology considered, it is observed that the instability wavelengths are governed by the streamwise and spanwise spacing between the roughness elements. In contrast, the roughness elements that are higher than the boundary layer create turbulent wakes in their lee. The scale of instability is much shorter and transition occurs due to the shedding from the obstacles. Post-transition, in the spatially developing TBL, the velocity defect profiles for both the smooth and rough walls collapsed when non dimensionalized in the outer units. However, when compared to the smooth wall, deviation in the Reynolds stresses are observable in the outer layer; the deviation being higher for the larger roughness elements.
\end{abstract}

Keywords Roughness · Transition - Secondary instability · Streaks · Turbulent boundary layer - Turbine blade

Nagabhushana Rao Vadlamani $(\bowtie)$, Paul G. Tucker

Department of Engineering, University of Cambridge, Cambridge, CB2 1PZ, UK

E-mail: nrv24@cam.ac.uk

Paul Durbin

Department of Aerospace Engineering, Iowa state university, Ames, Iowa, 50011, USA 


\section{Introduction}

Gas turbine engine manufacturers carefully monitor the surface finish of the components to be within the admissible tolerance levels. Such surfaces are considered to be 'aerodynamically smooth'. Once deployed into service, various damage mechanisms progressively degrade the surfaces of these components resulting in: a gradual drop in the performance of the engine, gradual increase in the specific fuel consumption, excessive temperatures in localized zones and under extreme cases the loss of stall margin [3]. During the design phase, aerodynamic analysis is carried out under the assumption that the surfaces are smooth; largely ignoring the aforementioned performance penalties (or in some cases benefits $[3,32]$ ) encountered due to surface roughness. In the literature, the studies concerning roughness can be broadly classified into the categories of: (a) roughness induced transition in a laminar boundary layer and (b) roughness enhanced turbulence in a turbulent boundary layer (TBL). A brief review of the relevant work is given here.

\subsection{Transitional boundary layers}

It is well known that the onset of the boundary layer transition is sensitive to the surface roughness. Post-transition, both the drag and heat transfer significantly increase over the aerodynamic surfaces. Reshotko [37] noted that smaller levels of roughness destabilize the mean flow through a linear amplification of the exponentially growing disturbances. On the other hand, larger levels of roughness bypass this route by distorting the flow locally [6, 28, 45]. Transition induced by isolated roughness has been extensively studied in the literature $[2,12,18,36]$, specifically at supersonic speeds. It was observed that the low-speed fluid behind the roughness element lifts up from the wall creating streamwise vortices. This is followed by a transient growth phenomena where an algebraic amplification of the initial disturbances evolve into streak like motions. In all the studies, an unstable detached shear layer is also shown to develop over the isolated roughness element which further accelerates transition to turbulence. A shear layer is also observed to develop on the distributed roughness elements, as shown in the recent direct numerical simulations (DNS) by Muppidi and Mahesh [29] at Mach $=2.9$. In their study, counter-rotating pairs of streamwise vortices developed underneath the shear layer. The interaction of these vortices with the shear layer is shown to trigger transition. The dataset generated from both the experimental and numerical studies are generally used to develop empirical correlations for predicting the transition onset $[2,36,39,45]$. Such correlations, when incorporated into the low order transition models, can be used to design efficient aerodynamic components. 
1.2 Turbulent boundary layers

The effect of roughness in fully turbulent boundary layers and channel flows has been addressed by several researchers starting from the classic work of Nikuradse [30]. In TBL, Townsend's [46] wall similarity hypothesis states that the turbulent motions outside the roughness sublayer are independent of the surface roughness. Jiménez [20] reviewed a variety of roughness topologies investigated by several researchers and noted that the dimensionless parameters; Roughness Reynolds number $k_{s}^{+}=k u_{\tau} / \nu$ and blockage ratio $\delta / k$; control the effect of roughness on TBLs. Surface topology of the rough surfaces in both the experimental and numerical investigations has evolved from idealized 2D elements $[24]$ to distributed $3 \mathrm{D}$ elements $[8,10]$ and more recently to the real rough surfaces $[25,51]$. Majority of the numerical investigations are confined to channel flows where periodicity is imposed in the streamwise direction. Lee et. al. [21] and Lee and Sung [22] simulated a spatially developing TBL and compared the effects of $2 \mathrm{D}$ rod-roughened and $3 \mathrm{D}$ cube-roughened walls. For both these configurations, surface roughness affected the turbulent Reynolds stresses in the outer layer.

From numerical perspective, it is evident that most of the eddy resolving simulations in literature have addressed transition induced by isolated roughness in supersonic flows. The objective of the present study is to investigate the effect of distributed roughness on subsonic boundary layers typically observed in turbomachines. However, unlike the recent work [32, 33, 42, 43, 47] where a flat plate has been subjected to turbine blade loading which triggered flow separation, current simulations are much more fundamental and consider a flat pate in the absence of pressure gradients. In contrast to the simulations of Muppidi and Mahesh [29], the rough surface is specified over the entire length of the flat plate. The motivation for such an arrangement is to cover the transition behaviour over a wide range of Reynolds numbers based on roughness height. Hence, once the flow undergoes transition, the current distribution also helps to explore the effects of surface roughness on the spatially developing TBLs and further validate the numerical framework.

The paper is organized as follows: computational framework, numerical algorithm and grid sensitivity study is described in the next section. In Section 3.1 , the effect of surface roughness on transition is explored where comparisons are made against the transition onset correlations. In Section 3.2, the transition mechanisms are investigated in detail. Section 3.3 presents the roughness effects on the spatially developing turbulent boundary layers and concluding remarks are provided in Section 4. 


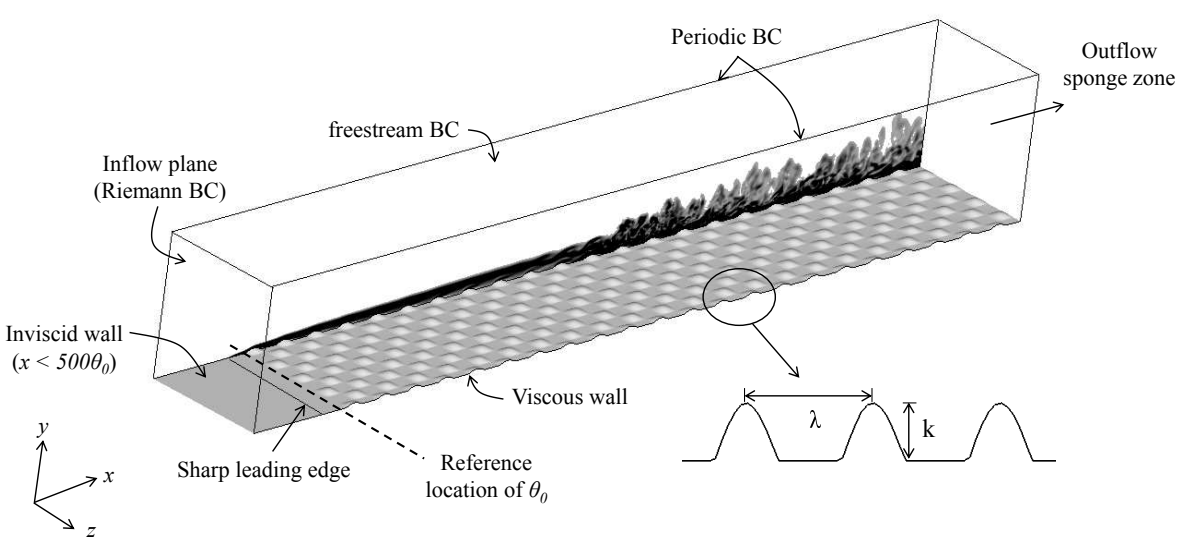

Fig. 1: Compuational domain and boundary conditions. Inset plot shows the roughness characteristics. Contours of vorticity magnitude on $x y$-plane demonstrate the transition of boundary layer.

\section{Numerical Framework}

\subsection{Computational domain and boundary conditions}

Figure 1 is a schematic of the computational domain and the associated boundary conditions. The numerical setup consists of a new boundary layer developing over the flat plate with a sharp leading edge. The flat plate extends for $L_{x}=10000 \theta_{0}, L_{y}=1000 \theta_{0}$ and $L_{z}=700 \theta_{0}$ in the streamwise, wall-normal and spanwise directions respectively. Here, $\theta_{0}$ is the momentum thickness of the laminar boundary layer developing on a smooth wall estimated at an axial location of $x=600 \theta_{0}$. This location corresponds to the leading edge of the first roughness element which is placed at a distance of $100 \theta_{0}$ from the leading edge of the flat plate. On a smooth wall, the momentum thickness Reynolds number $R e_{\theta_{0}}$ at this location is around 50. The free-stream Mach number is set to 0.5 .

A free-slip boundary condition is applied on the lower wall upstream of the leading edge $\left(x<500 \theta_{0}\right)$ while a no-slip boundary condition is imposed beyond $x \geq 500 \theta_{0}$. Free-stream boundary conditions are applied on the top boundary. Periodic boundary conditions are employed in the spanwise direction with a six point grid overlap to maintain the spatial accuracy. A non-reflective boundary condition based on Riemann invariants is imposed at the inflow as described in Matsuura and Kato [27]. In addition, following the approach of Rizzetta and Visbal [38], any spurious reflections are avoided by stretching the mesh beyond $x \geq 10000 \theta_{0}$ at the outlet and on the upper boundary. This method transfers information to higher wave numbers which is subsequently dissipated using a low pass filter [49]. 
The time-step is adjusted such that the maximum local CFL is 1.0 [27] which results in a non-dimensional time step of $0.1 \theta_{0} / U_{\infty}, U_{\infty}$ being the freestream velocity. After flushing out the initial transience, statistics are sampled on the fly over a non-dimensional time of $100,000 \theta_{0} / U_{\infty}$. This corresponds to around 10 charateristic time units based upon freestream conditions and the streamwise extent of the domain. Based on the local boundary layer thickness $\delta$ and friction velocity $U_{\tau}$, the sampling time of the statistics correspond to around $28 \delta / U_{\tau}$ at $R e_{\theta}=1000$. Statistical convergence of the mean quantities and Reynolds stresses are within $1 \%$.

Following [29], the surface roughness is defined as:

$$
\begin{gathered}
y_{\text {wall }}=\max \left(0, k \sin \left(k_{x} x\right) \sin \left(k_{z} z\right)\right) \quad \text { if } \quad x \geq 600 \theta_{0} \\
y_{\text {wall }}=0 \quad \text { elsewhere, }
\end{gathered}
$$

where $k$ is the peak amplitude of the roughness element, $k_{x}=2 \pi / \lambda_{x}$ and $k_{z}=2 \pi / \lambda_{z}$ are the wavenumbers in the streamwise and spanwise directions respectively. $\lambda_{x}$ and $\lambda_{z}$ are the corresponding wavelengths which are fixed at $200 \theta_{0}$ and $175 \theta_{0}$ such that roughness spans four full wavelengths in the spanwise direction. Observe that only the positive peaks of roughness elements are considered in the simulations while the negative troughs are clipped. Three different test cases with increasing amplitudes of roughness are considered $k=$ $4 \theta_{0}, 8 \theta_{0}, 16 \theta_{0}$ which will be henceforth referred as $k 4, k 8$ and $k 16$ respectively. The ratio between the streamwise wavelength to roughness height for these test cases are $\lambda_{x} / k=50,25,12.5$. Hence, in an engineering context, the surface configurations considered in this study can be classified as ' $\mathrm{k}$ ' type [23].

\subsection{Governing equations and numerical method}

Unsteady three dimensional compressible Navier-Stokes equations governing the fluid flow are transformed into the generalized curvilinear coordinate system. Following are the resulting governing equations in the conservative form:

$\frac{\partial \mathbf{Q}}{\partial t}+\frac{\partial}{\partial \xi_{1}}\left(\mathbf{F}_{i n v}-\frac{\mathbf{F}_{v i s}}{R e}\right)+\frac{\partial}{\partial \xi_{2}}\left(\mathbf{G}_{i n v}-\frac{\mathbf{G}_{v i s}}{R e}\right)+\frac{\partial}{\partial \xi_{3}}\left(\mathbf{H}_{i n v}-\frac{\mathbf{H}_{v i s}}{R e}\right)=0$

Here $t$ is time, $\xi_{1}, \xi_{2}, \xi_{3}$ are the transformed coordinates ${ }^{1}, \mathbf{Q}$ is the dependent variable vector, $\mathbf{F}_{i n v}, \mathbf{G}_{i n v}, \mathbf{H}_{i n v}$ are the inviscid flux vectors and $\mathbf{F}_{\text {vis }}$, $\mathbf{G}_{v i s}, \mathbf{H}_{v i s}$ are the viscous flux vectors. Following Visbal and Gaitonde [50], these vectors are given by:

1 Note that in some publications, $\left(\xi_{1}, \xi_{2}, \xi_{3}\right)$ are represented as $(\xi, \eta, \zeta)$ and $\left(x_{1}, x_{2}, x_{3}\right)$ as $(x, y, z)$ 


$$
\begin{aligned}
& \mathbf{Q}=\left(\begin{array}{c}
\rho \\
\rho u \\
\rho v \\
\rho w \\
E
\end{array}\right) \\
& \mathbf{F}_{i n v}=\left(\begin{array}{c}
\rho U \\
\rho u U+\xi_{1 x_{1}} p \\
\rho v U+\xi_{1 x_{2}} p \\
\rho w U+\xi_{1 x_{3}} p \\
(\rho E+p) U
\end{array}\right) \quad \mathbf{G}_{i n v}=\left(\begin{array}{c}
\rho V \\
\rho u V+\xi_{2 x_{1}} p \\
\rho v V+\xi_{2 x_{2}} p \\
\rho w V+\xi_{2 x_{3}} p \\
(\rho E+p) V
\end{array}\right) \\
& \mathbf{H}_{i n v}=\left(\begin{array}{c}
\rho W \\
\rho u W+\xi_{3 x_{1}} p \\
\rho v W+\xi_{3 x_{2}} p \\
\rho w W+\xi_{3 x_{3}} p \\
(\rho E+p) W
\end{array}\right) \mathbf{F}_{v i s}=\left(\begin{array}{c}
0 \\
\xi_{1 x_{i}} \tau_{i 1} \\
\xi_{1 x_{i}} \tau_{i 2} \\
\xi_{1 x_{i}} \tau_{i 3} \\
\xi_{1 x_{i}}\left(u_{j} \tau_{i j}-Q_{i}\right)
\end{array}\right) \\
& \mathbf{G}_{v i s}=\left(\begin{array}{c}
0 \\
\xi_{2 x_{i}} \tau_{i 1} \\
\xi_{2 x_{i}} \tau_{i 2} \\
\xi_{2 x_{i}} \tau_{i 3} \\
\xi_{2 x_{i}}\left(u_{j} \tau_{i j}-Q_{i}\right)
\end{array}\right) \quad \mathbf{H}_{v i s}=\left(\begin{array}{c}
0 \\
\xi_{3 x_{i}} \tau_{i 1} \\
\xi_{3 x_{i}} \tau_{i 2} \\
\xi_{3 x_{i}} \tau_{i 3} \\
\xi_{3 x_{i}}\left(u_{j} \tau_{i j}-Q_{i}\right)
\end{array}\right)
\end{aligned}
$$

where stress tensor $\tau_{i j}$, heat flux vector $Q_{i}$ and energy flux $E$ are given by:

$$
\begin{gathered}
\tau_{i j}=\mu\left(\frac{\partial \xi_{k}}{\partial x_{j}} \frac{\partial u_{i}}{\partial \xi_{k}}+\frac{\partial \xi_{k}}{\partial x_{i}} \frac{\partial u_{j}}{\partial \xi_{k}}-\frac{2}{3} \delta_{i j} \frac{\partial \xi_{l}}{\partial x_{k}} \frac{\partial u_{k}}{\partial \xi_{l}}\right) \\
Q_{i}=-\frac{\mu}{(\gamma-1) \operatorname{Pr} M_{\infty}^{2}} \frac{\partial \xi_{l}}{\partial x_{i}} \frac{\partial T}{\partial \xi_{l}} \\
E=\frac{T}{\gamma(\gamma-1) M_{\infty}^{2}}+0.5\left(u^{2}+v^{2}+w^{2}\right)
\end{gathered}
$$

In these equations, $u, v, w$ are the cartesian components of velocity. Also, $\rho, p, T$ are the density, pressure and temperature respectively. $\delta_{i j}(=1$ if $i=j$ and $=0$ if $i \neq j)$ is the kronecker delta. The perfect gas law $(p=\rho R T)$ is used to close the system of equations, $R$ being the gas constant. Molecular viscosity $(\mu)$ is estimated using Sutherland's law. The specific heat ratio and the Prandtl number are set to 1.4 and 0.72 respectively. Finally, $U, V, W$ represent the contravariant velocity components given by:

$$
\begin{gathered}
U=\xi_{1 x_{1}} u+\xi_{1 x_{2}} v+\xi_{1 x_{3}} w \\
V=\xi_{2 x_{1}} u+\xi_{2 x_{2}} v+\xi_{2 x_{3}} w \\
W=\xi_{3 x_{1}} u+\xi_{3 x_{2}} v+\xi_{3 x_{3}} w
\end{gathered}
$$

The length and velocity scales in the preceeding equations are non-dimensionalized by the characteristic length $L$ and freestream velocity $U_{\infty}$. Density and pressure are normalized by $\rho_{\infty}$ and $\rho_{\infty} U_{\infty}^{2}$ respectively. 
Simulations presented here are performed using an in-house high-order structured code, COMP-SQUARE. The solver has been developed on the basis of the numerical algorithm described in $[15,50]$. For all the test cases investigated here, sixth-order tri-diagonal compact finite-difference schemes are used for the interior nodes to spatially discretize the metric terms, inviscid and viscous fluxes. Following [15, 27, 38], fourth order one-sided compact schemes are used at the boundary nodes. The metric terms are recast in the conservative form as described in [50]. This ensures that the metric cancellation errors, which can degrade the accuracy of the solution, are minimized on distorted meshes. Time integration is carried out using an explicit four stage fourth-order Runge-Kutta (RK) scheme. The numerical instabilities arising due to the non-dissipative nature of the high-order compact central difference approximation are eliminated by filtering the conservative variables. For this purpose, $10^{\text {th }}$ order low pass Pade-type non-dispersive spatial filter is employed at each RK stage. Filtering is sequentially applied in each of the three computational directions. In the filter formulation, the adjustable parameter $\alpha_{f}$ $(-0.5<0 \leq 0.5)$ dictates the amount of filtering applied (see [50]). Higher values of $\alpha_{f}$ correspond to a less dissipative filter. As noted in [27], $\alpha_{f}$ influences both the stability and accuracy of the solution. In the current simulations $\alpha_{f}=0.492$, maintaining both the stability and high accuracy of the numerical scheme. The code utilizes MPI message passing for parallelization on the distributed memory platforms.

The numerical framework has been validated on a number of canoncial test cases: Taylor green vortex, inviscid vortex convection, turbulent channel flows and boundary layers. It has also been used to investigate the transitional boundary layers separating under the influence of streamwise pressure gradients [42] and highly accelerated flows with strong streamline curvature over an intake lip [34].

\subsection{Grid sensitivity study}

The number of grid points in the streamwise, wall-normal and spanwise directions are 2020, 192 and 192 respectively. This results in a total of approximately $75 \mathrm{M}\left(\times 10^{6}\right)$ mesh nodes. On the test case $k 16$, considering the highest value of the friction velocity $u_{\tau}$ in the domain $\left(u_{\tau} / U_{\infty} \approx 0.08\right.$, see figure $\left.13(\mathrm{c})\right)$, the grid resolution is within the wall units of $\Delta x^{+}<20, \Delta y^{+}<0.6$ and $\Delta z^{+}<14$. A grid sensitivity study has also been carried out for the test case $k 8$. Table 1 provides the details of three different grids used for the study, comprising around $9 \mathrm{M}, 36 \mathrm{M}$ and $75 \mathrm{M}$ nodes. The maximum value of the time-averaged turbulence kinetic energy $\left(k_{\max }\right)$ at each streamwise station is extracted at the mid-plane of the computational domain. Figure 2(a) plots the streamwise evolution of $k_{\max }$ predicted on the different grids. A steep increase of the TKE indicates that the flow is undergoing transition to turbulence. Consistent results are observed for grids B and C. The transition is delayed on grid A. This is most likely due to an insufficient resolution in the spanwise direction 


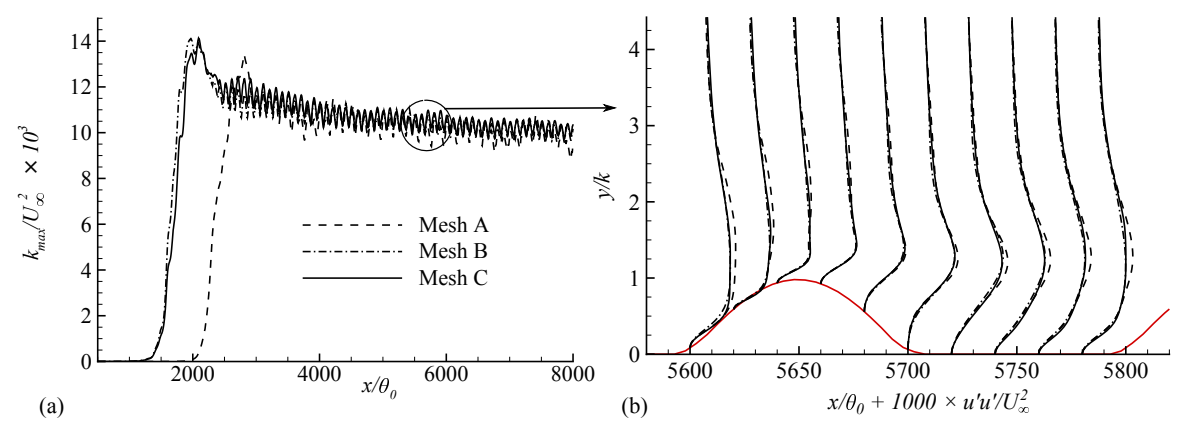

Fig. 2: Grid sensitivity study: (a) Streamwise evolution of the maximum value of the time-averaged turbulent kinetic energy (TKE) along the mid plane of the computational domain for test case $k 8$ (b) Carpet plot showing the profiles of $u^{\prime} u^{\prime}$ over a roughness element in the turbulent regime marked in figure (a).

Table 1: Computational parameters for grid sensitivity study

\begin{tabular}{cccc}
\hline \hline Grid & $N_{x}$ & $N_{y}$ & $N_{z}$ \\
\hline A & 1020 & 96 & 96 \\
B & 2020 & 96 & 192 \\
C & 2020 & 192 & 192 \\
\hline \hline
\end{tabular}

to capture secondary instabilities. Wall-normal profiles of $u^{\prime} u^{\prime}$ are also shown in figure 2(b). The profiles were extracted over a roughness element in the turbulent regime at an axial location $\left(5600 \leq x / \theta_{0} \leq 5800\right)$ indicated by an enclosed region in figure 2(a). Again deviations are noticeable on the coarsest grid A. However, consistent results are observed for grids $\mathrm{B}$ and $\mathrm{C}$ indicating that the results are grid independent. Despite achieving the convergence on grid $\mathrm{B}$ with $36 \mathrm{M}$ nodes, results presented in this manuscript are extracted from the finest mesh $\mathrm{C}$.

\section{Results and Discussion}

\subsection{Transitional Regime}

\subsubsection{Influence of increasing roughness height}

Roughness past an isolated roughness element has been extensively studied in the literature. The key characteristics governing the transition process in the current study with distributed roughness are similar to those observed over an isolated roughness element. Figure 3 compares the height of the roughness elements against the thickness of an unperturbed laminar boundary layer developing over the flat plate. While the roughness elements in the first two cases $k 4$ and $k 8$ are submerged within the boundary layer, the roughness element 


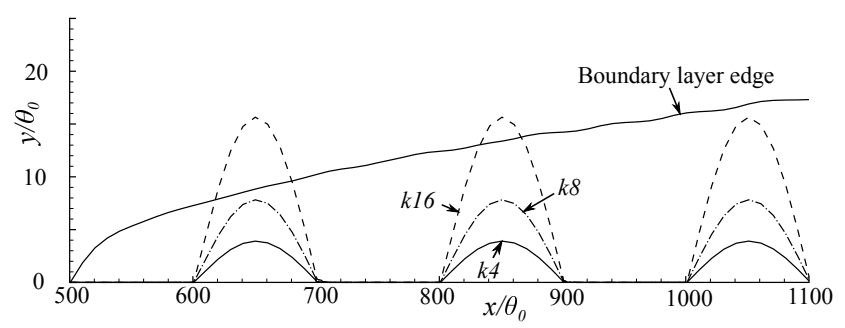

Fig. 3: Roughness elements embedded within and protruding into the boundary layer.

in $k 16$ case protudes beyond the boundary layer. Boundary layer thickness, $\delta$, at an axial location corresponding to the peak of the first roughness element is around $9 \theta_{0}$. Hence $k / \delta$ for the three test cases $k 4, k 8, k 16$ are around 0.44 , 0.88 and 1.76 respectively.

Local Reynolds number based on the roughness height $R e_{k}$ is a critical parameter to determine if the flow transitions to turbulence. $R e_{k}$ is defined as

$$
R e_{k}=\frac{\rho_{k} U_{k} k}{\mu_{k}}
$$

where $k, \rho_{k}, U_{k}$ and $\mu_{k}$ are the height of roughness element, fluid density, streamwise velocity and dynamic viscosity at height $k$ in a laminar boundary layer in the absence of roughness [36]. Based on this, $R e_{k}$ for the test cases $k 4, k 8$ and $k 16$ are estimated as 150,400 and 800 respectively at the peak of the first roughness element.

The effect of increasing the roughness height (and hence the roughness Reynolds number, $R e_{k}$ ) on transition can be visualized in Figure 4. Instantaneous contours of the vorticity magnitude on $x-z$ plane are shown in figure 4(a) at a wall-normal height of $y / \theta_{0}=20$. Contrasting trends in the transition behaviour are observable. For the test case $k 4$ with the lowest $R e_{k}=150$, the flow has not transitioned over the entire length of the flat plate. On the other hand, the flow has transitioned downstream at an axial location of $x / \theta_{0} \approx 1500$ for the case $k 8$ with $R e_{k}=400$. Consistent with the observation of Choudhari and Fischer [9], spontaneous transition occurs behind the first roughness element for the $k 16$ case with highest $R e_{k}$ of 800 . Note that the scale of the instability is much shorter in $k 16$ than $k 8$. The former is due to shedding from the obstacles; the latter is due to the instability of streaks.

Figure 4(b) compares the contours of vorticity magnitude on $x-y$ plane extracted at the mid-plane of the computational domain. A detached shear layer lifting away from the roughness elements is clearly seen in case $k 8$. The strength of the shear layer increases with $R e_{k}$. This was also observed by Redford et. al. [36]. The corresponding wall-normal distance by which the shear layer lifts away from the wall also increased with $R e_{k}$. For the test case $k 8$, the shear layer starts to break up at a streamwise location of $x / \theta_{0} \approx 1500$. At this station, Kelvin-Helmholtz (KH) type roll-up can be observed due to 


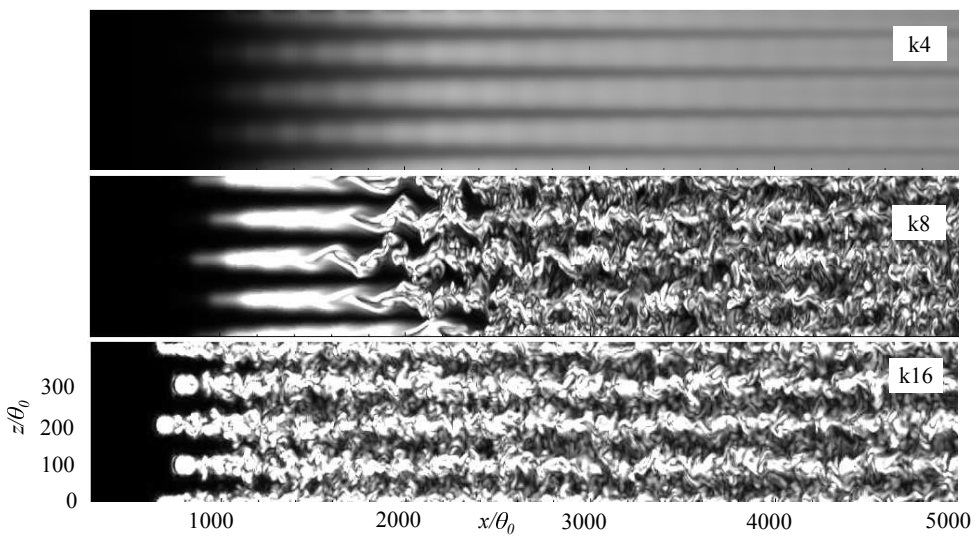

(a) On a wall normal height of $y / \theta_{0}=20$.
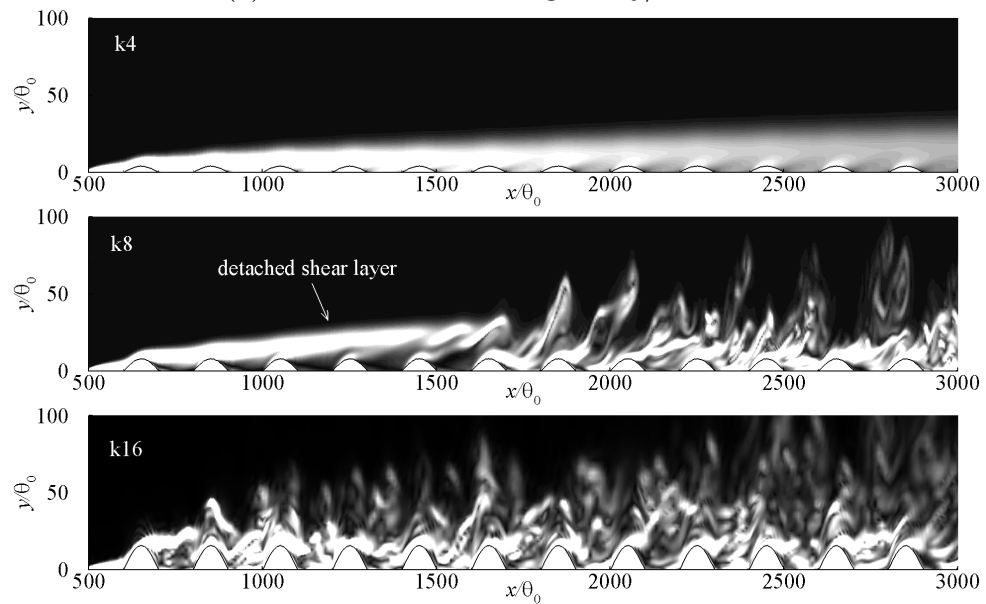

(b) On the mid-plane of the computational domain ( $y$ coordinate is stretched to show the lift-up of shear layer)

Fig. 4: Instantaneous contours of vorticity magnitude on $x-z$ and $x-y$ planes.

the intrinsic instability associated with the inflectional velocity profiles within the shear layer $[2,14,29]$. Detailed discussion on the transition mechanism for case $k 8$ will be presented in section 3.2 .

\subsubsection{Validation against transition onset correlations}

In this section, the validity of the transition behaviour observed in the preceeding section is verified by comparing against a set of correlations proposed in the literature. For this purpose, boundary layer integral parameters will be first presented. These parameters are estimated from a time averaged flow field. Spatial averaging has been carried out across the span at the same wall- 

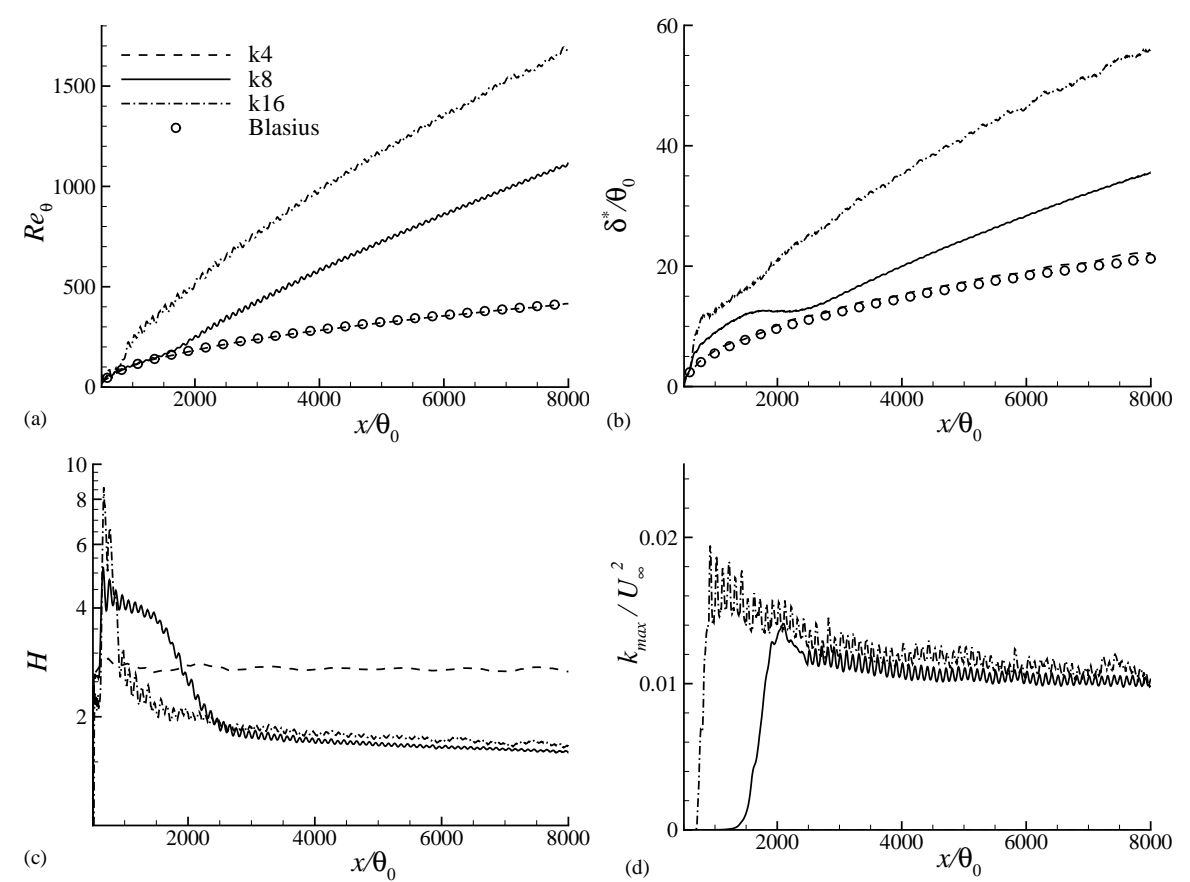

Fig. 5: Variations of (a) momentum thickness Reynolds number, $R e_{\theta}$, (b) displacement thickness, $\delta^{*}$ (c) Shape factor, $H$ and (d) maximum turbulent kinetic energy along $z-y$ plane, as a function of streamwise length with roughness height.

normal location $y$ from a zero-datum plane. It should however be noted that the flow is not homogeneous in space due to surface roughness i.e. the timeaveraged statistics are not independent of the location at a given wall-normal height.

Figure $5(\mathrm{a}, \mathrm{b}, \mathrm{c})$ shows the variation of the momentum thickness Reynolds number $\left(R e_{\theta}\right)$, displacement thickness $\left(\delta^{*}\right)$ and shape factor $(H)$ along the streamwise direction. Since the flow does not transition for the test case $k 4$, the variation of $\theta$ and $\delta^{*}$ are largely in agreement with the laminar Blasius solution. Due to the presence of roughness, a marginal increase in $\delta^{*}$ when compared to Blasius solution can be observed. Once the flow undergoes transition, $R e_{\theta}$ and $\delta^{*}$ increase almost steadily along the streamwise direction for the test cases $k 8$ and $k 16$, the increase being more pronounced for the $k 16$ case with the largest roughness. Figure 5(c) shows a significant increase of the shape factor, $H$ before the flow breaks down to turbulence. The shape factor converged downstream after transition, the values being again higher for the $k 16$ case when compared to $k 8$. The trends are in agreement with those observed by Lee and Sung [22] who compared the growth of a turbulent boundary layer on a rod roughned wall against that over a smooth wall. The streamwise variation 
of the maximum turbulent kinetic energy (TKE) is shown in Figure 5(d). A steep increase in the maximum TKE is notable during transition. For case $k 8$, the initial growth is algebraic which is in agreement with the transient growth phenomenon. Subsequently, the algebraic growth is overtaken by the exponential growth associated with $\mathrm{KH}$ instability. On the other hand, the TKE growth is quite abrupt for the case $k 16$, the laminar boundary layer being tripped by the first roughness element itself [9].

Stripf et. al. [45] investigated around 30 different configurations of rough surfaces. These included both regular distributions of truncated cones and random roughness. Measurements were collected at several Reynolds numbers and free-stream turbulence intensities. Based on this data, the following transition onset correlation for the rough surface was proposed:

$$
\begin{aligned}
R e_{\theta, t} & =R e_{\theta, t, s} \quad \text { if } \quad k / \delta_{t}^{*} \leq 0.01 \\
& =\left[\frac{1}{R e_{\theta, t, s}}+0.0061 . f_{\Lambda} \cdot\left(\frac{k}{\delta_{t}^{*}}-0.01\right)^{f_{T u}}\right]^{-1} \quad \text { if } \quad k / \delta^{*}>0.01
\end{aligned}
$$

Here $\operatorname{Re}_{\theta, t}$ and $\delta_{t}^{*}$ are the momentum thickness Reynolds number and displacement thickness estimated at the transition location for the rough surface. $R e_{\theta, t, s}$ is the momentum thickness Reynolds number at the transition location on a smooth surface which is given by $500 T u_{e f f}^{-0.75}$; where $T u_{e f f}$ is the effective turbulence intensity evaluated as an average between the $T u$ of the approacing flow and the local value of $T u$ at the boundary layer edge. $f_{\Lambda}$ is the roughness spacing parameter which characterizes the roughness density. It is a function of $\Lambda_{R}=k / h_{m}$, where $h_{m}$ is the mean elevation height of the roughness topography. For the roughness topologies considered in the current study $\Lambda_{R}=5$ and $f_{\Lambda} \approx 1$ based on the recommended values of Stripf et. al. [45]. In Eq. 11, $f_{T u}$ is a function of $T u_{e f f}$ given by:

$$
f_{T u}=\max \left[0.9,1.61-1.15 e^{-T u_{e f f}}\right]
$$

In the current simulations, the values of $R e_{\theta, t}$ and $k / \delta_{t}^{*}$ have been extracted at an axial location corresponding to a notable increase in the TKE. This is evident from the figure 5(d) and indicates the onset of flow transition. Figure 6(a) overlays the current predictions against the correlation proposed by Stripf et. al. [45]. The present results are in good agreement with their experimental correlation. Note that the simulations addressed in this manuscript do not include any FST at the inlet ${ }^{2}$. Hence the correlation has been estimated under the limiting condition of $T u_{e f f}=0$ as follows:

\footnotetext{
2 We have also carried out additional simulations to explore the effects of free-stream turbulence (FST). However, this is beyond the scope of the current paper and will be published elsewhere.
} 

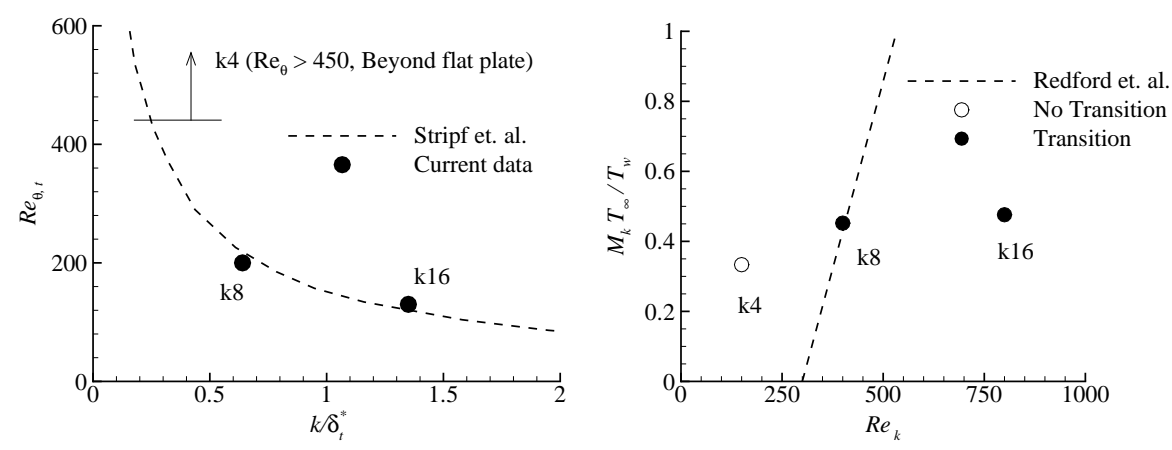

Fig. 6: Comparison of the transition onset on rough surfaces from the current simulations against the (a) correlation proposed by Stripf et. al. [45] on $R e_{\theta, t}$ $\delta_{t}^{*}$ plane and (b) correlation proposed by Redford et. al. [36] on $M_{k} T_{\infty} / T_{w}-$ $R e_{k}$ plane.

$$
R e_{\theta, t}=\left[0.0061 . f_{\Lambda} \cdot\left(\frac{k}{\delta_{t}^{*}}-0.01\right)^{0.9}\right]^{-1} \quad \text { if } k / \delta^{*}>0.01
$$

Notice that under this limit, $f_{T u}=0.9$ and the transition onset Reynolds number is primarily governed by the second term in Eq. 11, while the contribution from the first term $\left(1 / R e_{\theta, t, s}\right)$ vanishes. For the test case $k 4, k / \delta^{*}$ at the exit of the domain is around 0.05 . At this location, the correlation suggests that $R e_{\theta, t}$ should be $\approx 1000$ for the flow to undergo transition. However, it is evident from figure 5(a) that $R e_{\theta}$ at the exit for the test case $k 4$ is around 450 which is substantially lower than the threshold value. Hence the observation that the flow has not transitioned on the flat plate for the test case $k 4$ is consistent with the expected behaviour from the experimental correlation.

Redford et. al. [36] carried out simulations on an isolated roughness element over a range of Mach numbers and proposed a criterion for the transition behaviour. It is based on the Mach number at the edge of the roughness element $\left(M_{k}\right), R e_{k}$ and the ratio of free-stream to wall temperatures $\left(T_{\infty} / T_{w}\right)$. The dividing line that separates the laminar and transitional cases is given by:

$$
\frac{M_{k} T_{\infty}}{T_{w}}=\frac{3\left(R e_{k}-300\right)}{700}
$$

$M_{k}$ and $R e_{k}$ from the present computations are estimated at the peak of the first roughness element. Figure 6(b) compares the estimated values against the criterion proposed by Redford et. al. [36]. Interestingly, the current results, with distributed roughness, compare favourably against their criterion of isolated roughness. Notice that test case $k 8$ is almost at the threshold that demarks the laminar and transitional regimes. However, given that the roughness element in the current study is placed close to the leading edge of the 


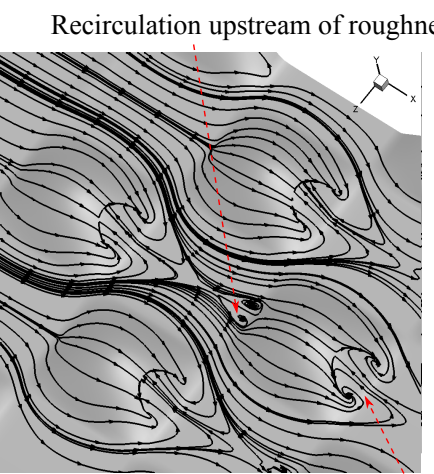

(a) $\mathrm{k} 8$ saddle points

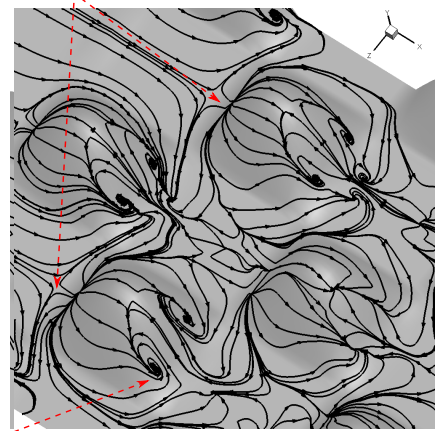

(b) k16

Recirculation behind roughness elements

Fig. 7: Comparison of the surface streamlines for test cases $k 8$ and $k 16$.

flat plate, $k / \delta$ for this test case is around 0.8 . This is greater than the value of $k / \delta=0.65$ used by Redford et. al. and hence is more likely to trigger transition. As noted by Reda [35], it is expected that the transition onset over distributed roughness patterns occurs earlier when compared to the isolated roughness.

\subsection{Transition mechanism}

Figure $7(a, b)$ shows the surface streamlines over the roughness elements for the cases $k 8$ and $k 16$, respectively. It can be seen that the uniform laminar flow decelerates as it approaches the first roughness element. Part of the flow travels around it and rest of the flow is displaced away from the wall. Lift up of the near wall fluid results in the formation of a detached shear layer over the roughness element (see Figure 4(b)). A wake type flow forms behind the element and is characterized by the recirculation zones marked in figure 7. Subsequent roughness elements encounter a non-uniform wake flow from the preceeding ones resulting in a stronger deceleration. Observe from figure 7 (a) that the recirculation zone behind the second element is larger than the first. This leads to a decelerated flow in the line of roughness elements and an accelerated flow in between the roughness elements. This is similar to that observed by Muppidi and Mahesh [29]. For case $k 16$, recall that the roughness element penetrated through the boundary layer and shows clear evidence of the saddle points upstream of the roughness elements (see figure 7(b)). As expected, the recirculation behind the roughness elements is also stronger when compared to case $k 8$.

Progressive acceleration in between the roughness elements and deceleration in the line of the roughness elements result in the formation of steady streamwise streaks. Figure 8 shows iso-surfaces of the streamwise disturbance velocity, $u^{\prime}$. Alternating high $\left(u^{\prime}=0.2 U_{\infty}\right)$ and low $\left(u^{\prime}=-0.2 U_{\infty}\right)$ speed 


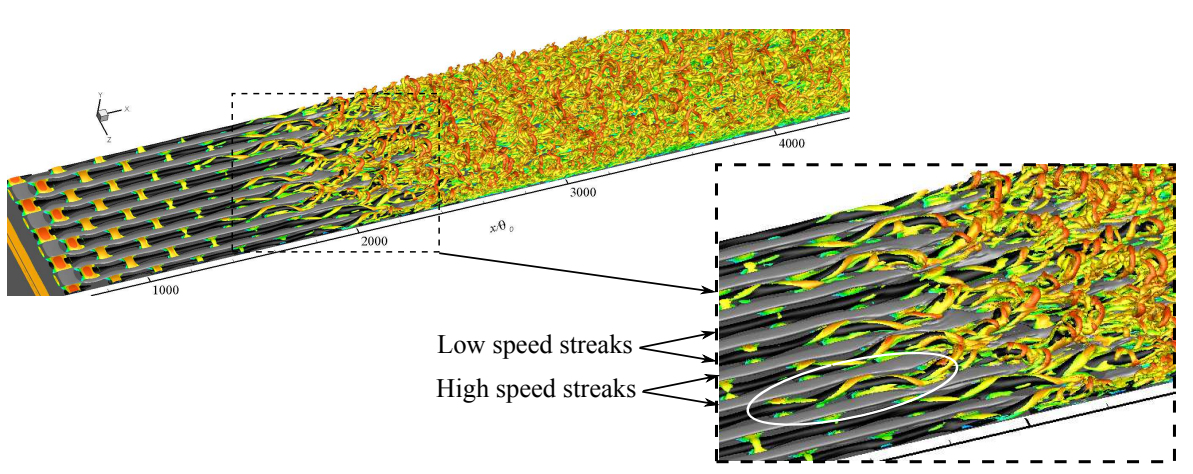

Fig. 8: Iso-surfaces of $\mathrm{Q}(=200)$ coloured with axial velocity for test case $k 8$. Iso-surfaces of streamwise velocity fluctuation $u^{\prime}$ are also overlaid. Positive perturbation $\left(u^{\prime}=0.2 U_{\infty}\right)$ represented by light grey and negative perturbation $\left(u^{\prime}=-0.2 U_{\infty}\right)$ indicated by black. Inset plot shows the magnified view of the transition zone and evolution of hairpin vortices.

streaks are indicated by the light and dark iso-surfaces. Vortical structures identified using iso-surfaces of $Q(=200)$, coloured with axial velocity, are also overlaid in the figure. The inset plot shows a magnified view of the transitional zone. Notice that the streamwise streaks are steady earlier on and subsequently become unsteady beyond $x / \theta_{0}>1500$. Beyond this station, secondary instabilities develop over the streaks; specifically the vortical structures appear on the flanks of the low-speed streaks. Hairpin vortices start originating at this point and a sustained turbulent flow develops downstream.

Secondary instability developing on one of the low-speed streaks marked in the inset plot of figure 8 is further analyzed. It should be noted that in order to elucidate the steady streamwise streaks, $u^{\prime}$ in the figure 8 is defined relative to the time and spanwise averaged mean flow i.e. $u_{x, y, z, t}^{\prime}=u_{x, y, z, t}-$ $\bar{u}_{x, y}$. While this approach is feasible above the roughness elements, it results in a perturbation within the roughness element. Instead, figure 9 shows the contours of streamwise fluctuations defined relative to the time-averaged flow field without any span-averaging or phase-averaging ${ }^{3}$ i.e. $u_{x, y, z, t}^{\prime}=u_{x, y, z, t}-$ $\bar{u}_{x, y, z}$. This will isolate the effects of the secondary instability developing on a steady streak. Figure 9 (a) shows the wall-parallel plane extracted at a height of $y / \theta_{0}=20$ and $9(\mathrm{~b})$ shows an $x-y$ plane extracted at the centreline of the streak $\left(z / \theta_{0}=430\right)$. Velocity vectors of the streamwise disturbance velocity $u^{\prime}$ and spanwise velocity $w$ are overlaid. Vortical structures identified by $Q$ are also shown using the dashed lines. Spanwise oscillations that are antisymmetric with respect to the centre line of the streak are evident on the low speed streak. This is reminiscent of the 'sinuous' type instability noted by several researchers in literature ( for example, see Brandt et. al. [5], Hack and Zaki [16])

\footnotetext{
3 In the case of regularly distributed roughness, $u^{\prime}$ can also be defined by subtracting the instantaneous flow from the flow field averaged over multiple roughness elements at identical phase. However, this approach is not feasible for randomly distributed roughness.
} 

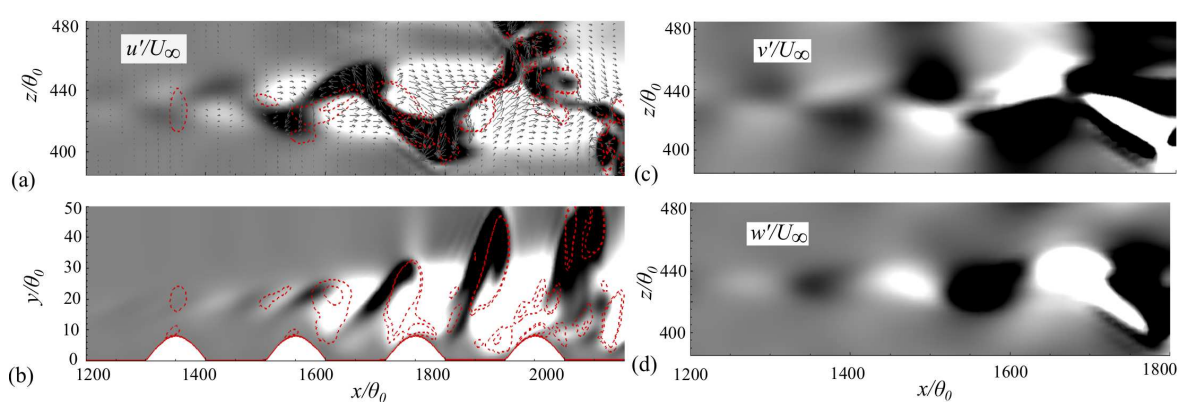

Fig. 9: Contours of disturbance velocity $u^{\prime}\left( \pm 0.2 U_{\infty}\right)$ showing sinuous like breakdown on (a) wall-parallel plane extracted at $y / \theta_{0}=20$ and (b) on $x-y$ plane extracted at the centreline of the streak $\left(z / \theta_{0}=430\right)$. Core of vortical structures is shown using dashed lines which mark regions of $Q=200$. Velocity vectors represent $u^{\prime}$ and spanwise velocity $w$. Contours of (c) wall-normal $\left(-0.025 \leq v^{\prime} / U_{\infty} \leq 0.025\right)$ and (d) spanwise $\left(-0.05 \leq w^{\prime} / U_{\infty} \leq 0.05\right)$ velocity fluctuations on wall-normal plane $y / \theta_{0}=20$.

in the context of transition due to free-stream turbulence. The velocity vectors show the intense shear between the high and low speed streaks and the local recirculation zones. Strong vortex cores (see dashed lines) are predominant in the region of this shear.

On the other hand, KH type rollers can be observed in figure 9(b). Recall from figure 4(b) (for test case $k 8$ ) that a detached shear layer develops over the roughness elements which destabilizes at $x / \theta_{0}=1500$. High and low speed streaks shown in figure 8 are beneath the shear layer. Secondary instabilities that develop on the low speed streak also grow at the axial location $x / \theta_{0}=1500$. This indicates that the secondary instability growing on the streaks underneath the shear layer influences the KH type shear layer instability observed in literature $[2,29,36]$ in the context of roughness induced transition. Transition to turbulence progresses under the combined influence of both these instabilities.

Figures 9(c,d) also show the contours of wall-normal $\left(v^{\prime}\right)$ and spanwise $\left(w^{\prime}\right)$ fluctuations. An antisymmetric pattern is evident in the contours of both $u^{\prime}$ and $v^{\prime}$, unlike $w^{\prime}$ which is symmetric with respect to the centreline of the low-speed streak. The magnitude of $w^{\prime}$ is also larger than that of $v^{\prime}$. Using linear stability analysis, Hack and Zaki [16] computed the eigenfunctions of the sinuous outer mode instability in the context of bypass transition due to free-stream turbulence. The patterns of the perturbation fields observed in the current study are in accordance with the shapes of the eigenfunctions computed by them (see figure 5 in [16]) using local stability approach. It is worth pointing out that, the global modes obtained from the recent global stability analysis by Loiseau et. al. [26] on an isolated cylindrical element and by Citro et. al. [11] on an isolated hemispherical roughness element are similar to the ones obtained using local stability approach. As noted by Loiseau et. 

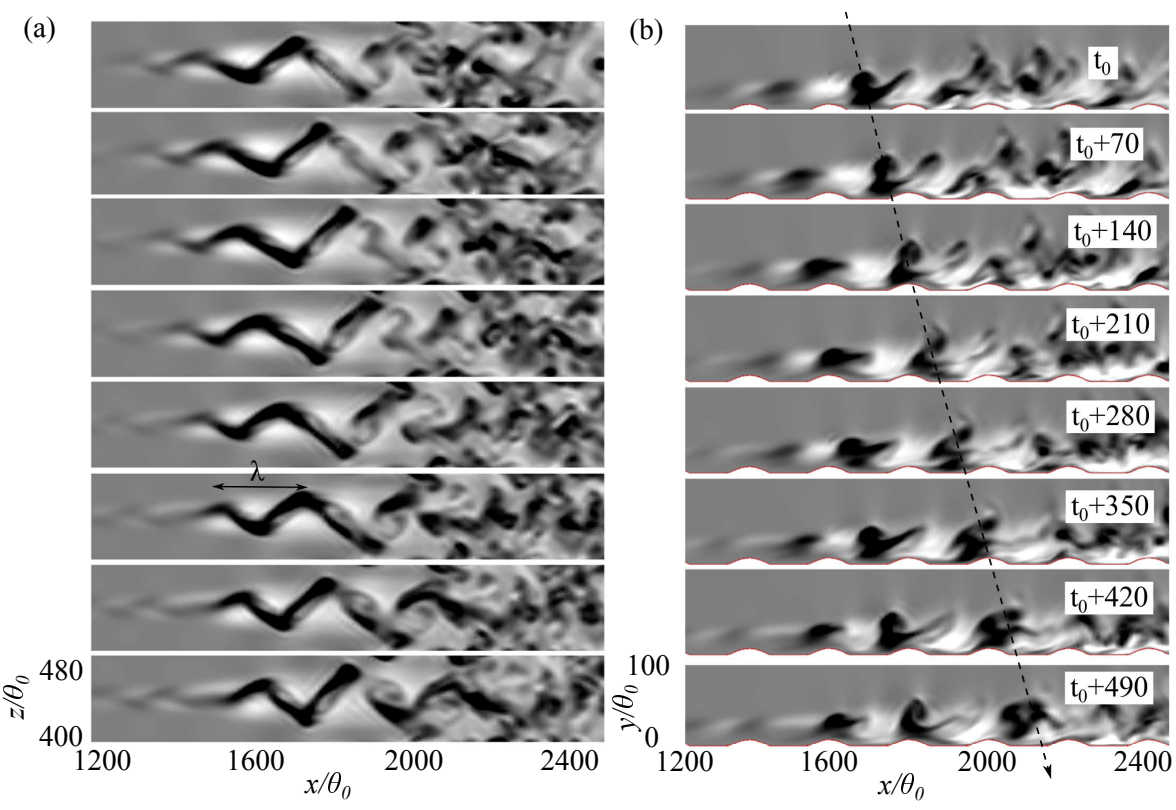

Fig. 10: Temporal evolution of the sinuous like breakdown on a low-speed streak on a (a) wall-parallel plane $\left(y / \theta_{0}=20\right)$ and (b) $x-y$ plane extracted at the centreline of the streak $\left(z / \theta_{0}=430\right)$. Contours show the disturbance velocity $u^{\prime}\left( \pm 0.2 U_{\infty}\right)$. Time interval between the pictures is $70 \theta_{0} / U_{\infty}$.

al. [26], the secondary instabilities appear relatively far from the roughness element where the base flow is nearly parallel with a dominant streamwise component. Hence both the global and local stability analysis give similar mode shapes. However, deviations are expected in the vicinity of the roughness element due to the non-parallel flow and hence the critical Reynolds number can be over or under predicted. It will be interesting to further investigate this aspect by carrying out global stability analysis of the current DNS results on distributed roughness elements.

Figure 10 further elucidates the secondary instability using a time sequence of sinuous type breakdown. Both wall-parallel and $x-y$ planes are shown in the figure. Notice that the streak is steady early on and the sinuous instability develops downstream. Spanwise antisymmetric oscillations can be visualized on the wall-parallel plane and the unsteady $\mathrm{KH}$ type shedding from the $x-y$ plane. Streaks are almost locked over the roughness elements for a prescribed roughness distribution. Hence there isn't any appreciable change in the transition location in space and time. This is indeed the case for all the streaks that can be seen in figure 8 . The streamwise wavelength of the sinuous instability is estimated to be around $200 \theta_{0}$. When non-dimensionalized with respect to the inlet boundary layer thickness used by Hack and Zaki [16], the corresponding wavelength is around $12.5 \delta_{0}$ which is in agreement with their values. Inter- 

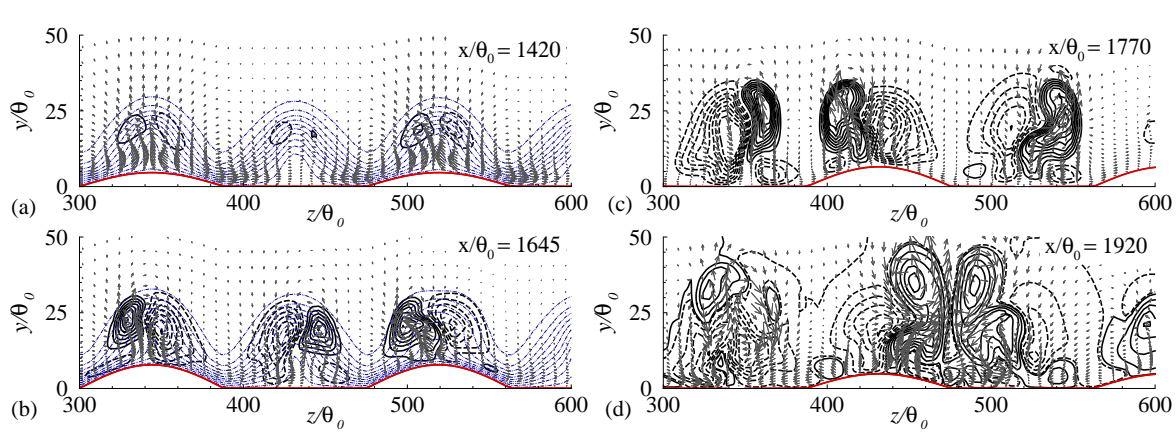

Fig. 11: $y-z$ planes extracted in the cross-stream direction at different axial locations (Solid lines: positive fluctuation, Dashed lines: negative fluctuation). Arrows indicate instantaneous velocity vectors of spanwise velocity $w$ and wallnormal velocity $v$. Frames (a) and (b) show the base flow using dashdot lines of constant streamwise velocity from 0.05 to 0.95 with a spacing of 0.1 .

estingly, in the current simulations, the streamwise spacing $\left(\lambda_{x}\right)$ between the roughness elements is also $200 \theta_{0}$. It will be worth verifying if the wavelength of secondary instability changes for a different topology with randomized roughness distribution. However, it is beyond the scope of the current study. The phase speed of the outer instability is estimated by tracking the extrema of $w^{\prime}$ in space and time. The phase speed is found to be $0.72 U_{\infty}$ which is close enough to the value of $0.78 U_{\infty}$ reported by Vaughan and Zaki [48] and Hack and Zaki [16]. This confirms that the secondary instability observed in the present computations is an outer sinuous mode rather than the inner modes which are characterized by much more smaller phase speeds $\left(\approx 0.3-0.4 U_{\infty}\right.$ [4]).

Cross-stream planes (or $y-z$ planes) are extracted at several streamwise locations between $1400 \leq x / \theta_{0} \leq 1950$ which covers around two wavelengths of the sinuous oscillation of the low speed streak shown in figure 9 . Instantaneous flow on these planes is presented in figure 11 using contours of streamwise velocity perturbation $u^{\prime}$. Frames (a,b) also show the base flow using the contours of streamwise velocity (dashdot lines). Velocity vectors based on the spanwise $w$ and wall-normal $v$ velocities are overlaid. It can be seen that the low-speed streaks (local streamwise velocity being lower than the span-averaged mean) are formed over the peaks of the roughness elements while the high-speed streaks (local streamwise velocity being higher than the span-averaged mean) are situated mid-way between the low speed streaks. The spanwise spacing between two low-speed streaks is around $80-85 \theta_{0}$ which is half of the spanwise spacing $\left(0.5 \lambda_{z}\right)$ between the roughness elements ${ }^{4}$. In terms of the wall-normal distance, the low-speed streaks are farther away from the wall compared to

\footnotetext{
4 Note: The low-speed streak situated mid-way between the roughness peaks in figure 11(a) is due to the peaks of the roughness element at an upstream location (refer to the computational domain in figure 1)
} 
the high-speed streaks. Frames (a) and (b) show that the streaks are initially coherent. Further downstream, the interaction between the high and low-speed streaks is clearly evident from frames (c) and (d) with a noticeable increase in $u^{\prime}$ across the junction between them. Intense shear between the streaks leads to oscillations in the spanwise direction and subsequently to secondary sinuous instability. In the context of roughness-induced transition on isolated roughness element, Denissen and White [13] also noted such secondary instabilities which are much more unstable in the mid-wake region than the far-wake region. More specifically, the instability observed in the current study is similar to the subharmonic sinuous mode observed by Andersson et. al. [1]. In frame (d), velocity vectors indicate the presence of strong counter rotating vortices at a wall-normal location of around $y / \theta_{0}=30$. The counter-rotating vortex pair accelerates the mixing process: low momentum fluid near the wall being pumped into the outer shear layer region and high momentum fluid being pushed closer to the wall. This eventually destabilizes the shear layer which has developed over the roughness elements initiating transition to turbulence [29].

\subsection{Turbulent regime}

In the current simulations, roughness elements are distributed over the entire surface of the flat plate (see figure 1). Once the flow undergoes transition and breaks down to turbulence, interaction between the roughness and the subsequent turbulent boundary layer can hence be explored, albeit at low Reynolds numbers. As mentioned in the introduction, the effect of surface roughness in turbulent channel flows has been extensively investigated in literature $[10,20,24]$. In the context of roughness effects on turbulent boundary layers, there are relatively fewer experimental [17,41] and numerical studies $[21,22]$. This section will delve into the effect of surface roughness on the mean and turbulent statistics of a spatially developing turbulent boundary layer.

In order to firstly demonstrate the accuracy of the current numerical framwork in the turbulent regime, an additional simulation has been carried out. The new test case (denoted by $k 8_{\text {new }}$ ) is a variant of $k 8$ and the roughness for this new test case is confined only to $600 \leq x / \theta_{0} \leq 2500$. Beyond $x / \theta_{0}>2500$, the flat plate is smooth. The flow has fully transitioned at $x / \theta_{0}=2500$ and a new turbulent boundary layer develops over the smooth surface. Figure 12(a,b) compares the current results against the DNS of Schlatter and Örlü [40]. Both the mean velocity profiles and the corresponding Reynolds stresses at two different axial locations correponding to $R e_{\theta}=670$ and $R e_{\theta}=1000$ are in encouraging agreement with that of the DNS.

\subsection{Skin friction and form drag}

The coefficient of skin friction $\left(C_{f}\right)$ and pressure drag (or form drag) $\left(P_{d}\right)$ are evaluated from the current simulations. These are given by $[24,31]$ : 

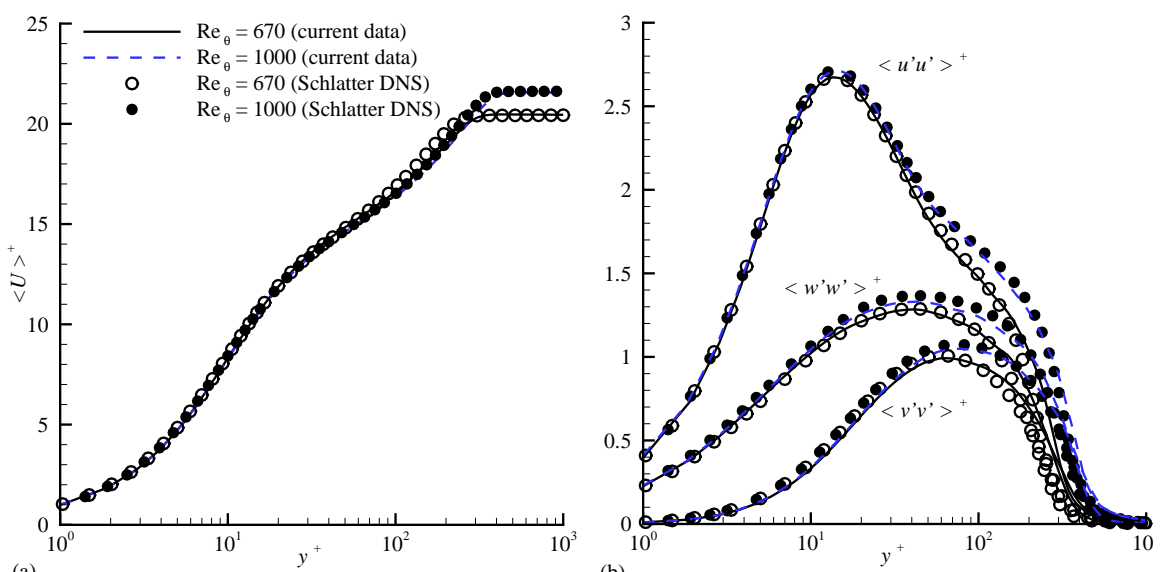

(a)

(b)

Fig. 12: Comparison against DNS by Schlatter and Örlü (a) Mean profiles and (b) turbulent Reynolds stresses at $R_{\theta}=670$ and $R e_{\theta}=1000$ for test case $k 8_{\text {new }}$

$$
C_{f}=\frac{1}{0.5 \rho U_{\infty}^{2} S_{s m}} \iint_{S} \mu\left(\frac{d u}{d n}\right)_{w} d S \quad P_{d}=\frac{1}{0.5 \rho U_{\infty}^{2} S_{s m}} \iint_{S} P_{w} n_{x} d S
$$

Here, $n_{x}$ is the streamwise component of the surface normal vector $n, P_{w}$ is the pressure evaluated at the wall, $S$ is the wetted area of the surface and $S_{s m}$ is the corresponding area of the smooth surface. Subsequently, friction velocity $\left(u_{\tau}\right)$ is estimated from the total drag given as $u_{\tau}=\left(0.5\left(C_{f}+P_{d}\right)\right)^{0.5}$. Note that $u_{\tau}$ has also been estimated using the constant stress layer suggested by Cardillo et. al. [7]. The values of $u_{\tau}$ obtained from both these approaches are found to be similar. Unlike in the channel flows, where periodic conditions are imposed in both the spanwise and streamwise directions, the characteristics of a spatially developing turbulent boundary layer evolve in the streamwise direction. The presence of 3D distributed roughness also introduces inhomogenity across the span. Following the approach of Lee et. al. [21], both $C_{f}$ and $P_{d}$ are hence spatially averaged over the surface $S$ spanning $\lambda_{x} \times \lambda_{z}$ i.e. over one roughness element. Also $u_{\tau}$ is evaluated based on the spatially averaged total drag.

Figure 13 shows the streamwise variation of $C_{f}, P_{d}$ and $u_{\tau}$ for all the three test cases. For comparison, $C_{f}$ for the laminar $\left(C_{f}=0.664 / R e_{x}^{0.5}\right)$ and turbulent boundary layers $\left(C_{f}=0.0592 / R e_{x}^{0.2}\right)$ on smooth surfaces are also overlaid in the figure 13(a). Recall that the flow remains laminar for case $k 4$. Hence the pressure drag is minimal and the skin friction coefficient agrees well with the laminar value for this case. For the cases $k 8$ and $k 16, C_{f}$ approaches close enough to the turbulent skin friction values on a smooth wall. However, due to the recirulations behind the roughness elements (see figure 7), the 

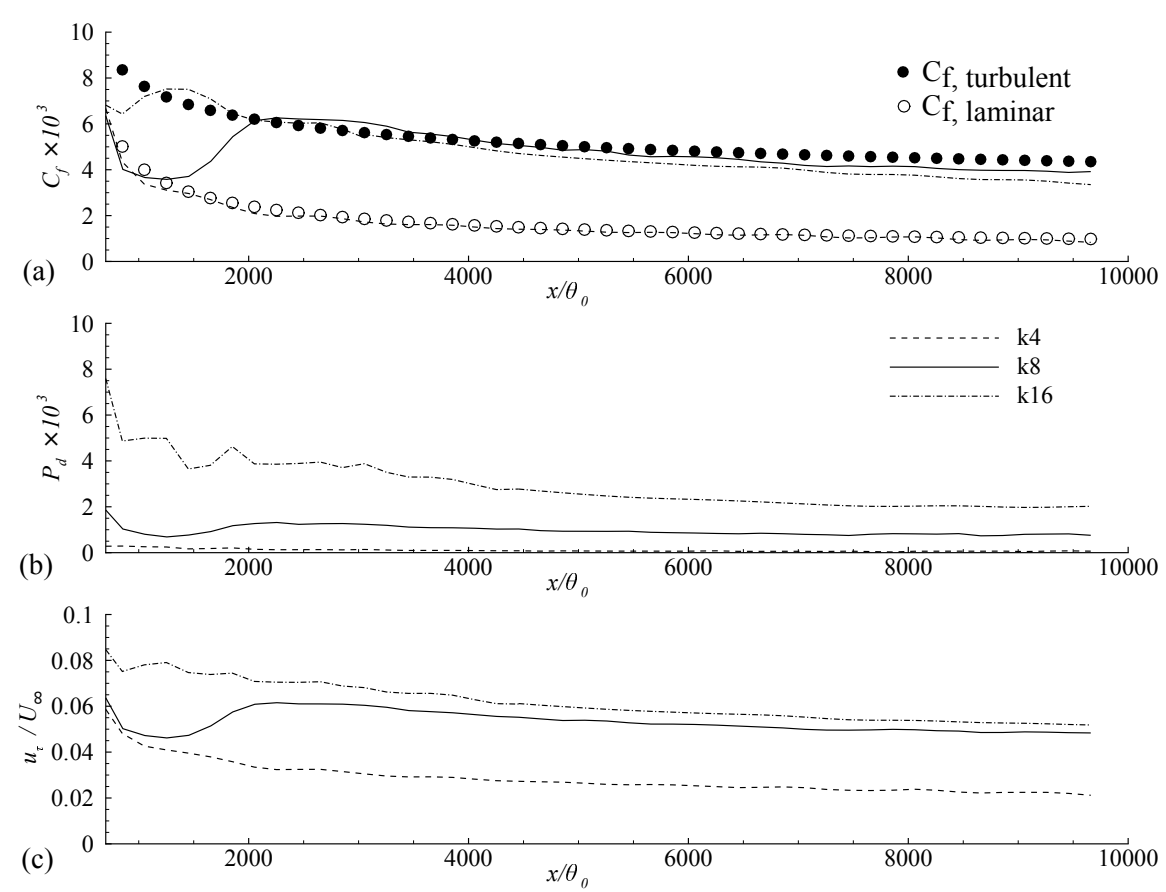

Fig. 13: Streamwise variation of (a) skin-friction coefficient, $C_{f}$ (b) pressure drag coefficient, $P_{d}$ and (c) friction velocity for cases $k 4, k 8$ and $k 16$.

spatial averaged skin friction is lower than that over a smooth wall. Due to larger recirculations, $C_{f}$ for the case $k 16$ is lower than that of $k 8$. In contrast, larger roughness elements induce a higher pressure drag. Hence, $P_{d}$ for the test case $k 16$ is higher when compared to $k 8$, thereby increasing the overall drag and the corresponding $u_{\tau}$.

\subsection{Roughness sublayer}

For the test case $k 8$, time-averaged contours of $u^{\prime} u^{\prime}$ and $-u^{\prime} v^{\prime}$ normalized by $u_{\tau}^{2}$ are shown in figure 14. $x_{r e f}$ represents the reference location of the leading edge of the roughness element of interest which is the chosen as $5640 \theta_{0}$. Streamwise velocity fluctuations increase above the crests of the roughness elements. A local maxima in $u^{\prime} u^{\prime}$ is observable at a wall-normal height of around $y / k=1.5$ and at an axial distance of around $10 k$ from the peak of the roughness elements. Consistent with the DNS results of Lee and Sung [21] on rod-roughened surfaces, there is a noticeable increase in the Reynolds shear stress above the cavity mid way between the roughness elements.

Profiles of mean velocity and Reynolds stresses are extracted at three different axial locations indicated by $L 1, L 2, L 3$ in the figure 15 . Evidently, the 
(a)

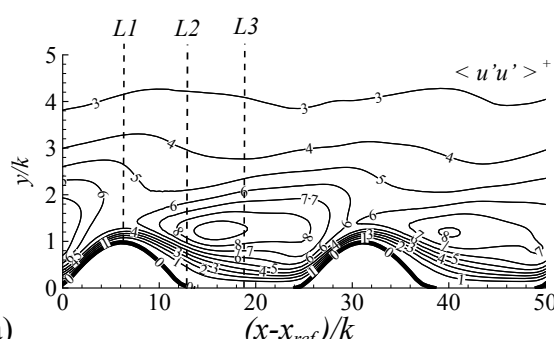

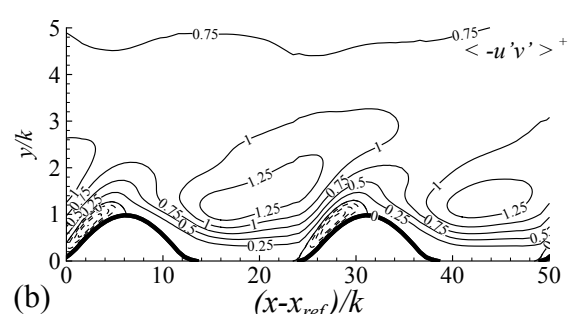

(b)

$\left(x-x_{r e f}\right) / k$

Fig. 14: Contours of (a) $u^{\prime} u^{\prime}$ and (b) $u^{\prime} v^{\prime}$ in the roughness sublayer $(y / k \leq 5)$ for case $k 8$ at $x_{r e f}=5640 \theta_{0}$.
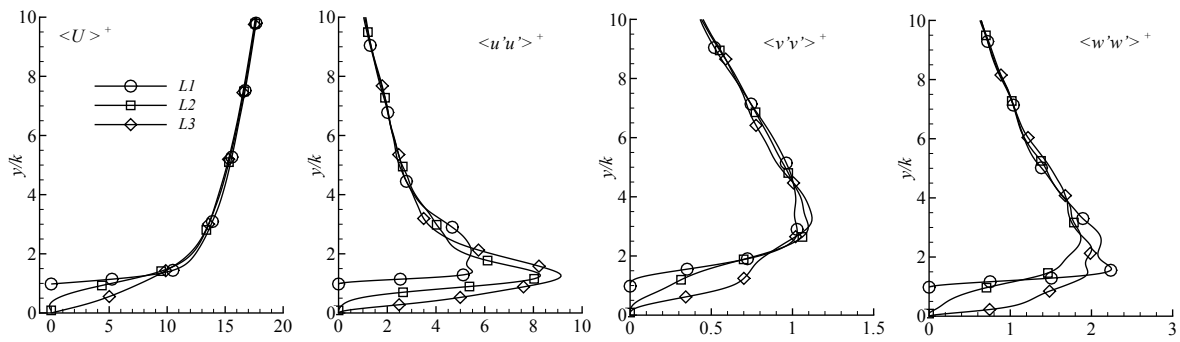

Fig. 15: Variations of mean velocities and turbulent Reynolds stresses at locations $L 1-L 3$ in the roughness sub-layer for test case $k 8$.

effect of roughness is confined within $y<4 k$ beyond which the profiles collapse, indicating that the roughness sublayer is within $y=4 k$. The results for the test case $k 16$ are similar to those of $k 8$, except that the roughness sublayer extends upto $y=6 k$.

\subsection{Roughness effects on mean velocity and Reynolds stresses}

From figure 13(c) it can be noted that the change in the friction velocity is marginal for both the cases $k 8$ and $k 16$ beyond $x / \theta_{0}>5000$. The thickness of the boundary layer also increases linearly downstream. Both these conditions are essential for the self-preservation in a rough-wall turbulent boundary layer $[22,44]$. Figure 16(a) compares the mean velocity profile for the test cases $k 8$ and $k 16$ against that of a smooth wall in inner variables. Note, $y^{+}$is defined as $y^{\prime} u_{\tau} / \nu$, where $y^{\prime}=y-\epsilon$ is the distance from the virtual origin. Here $\epsilon$, is the distance from the bottom wall to the virtual origin. It is estimated using the method proposed by Jackson, [19] evaluating the centroid of the moment of forces acting on the roughness elements. Beyond $x / \theta_{0}>5000, \epsilon / k$ for the test cases $k 8$ and $k 16$ are almost constant and are estimated to be around 0.31 and 0.29 respectively.

Following Lee and Sung [22], the profiles shown in figure 16 are extracted at an axial location where the boundary layer thickness is identical for both 

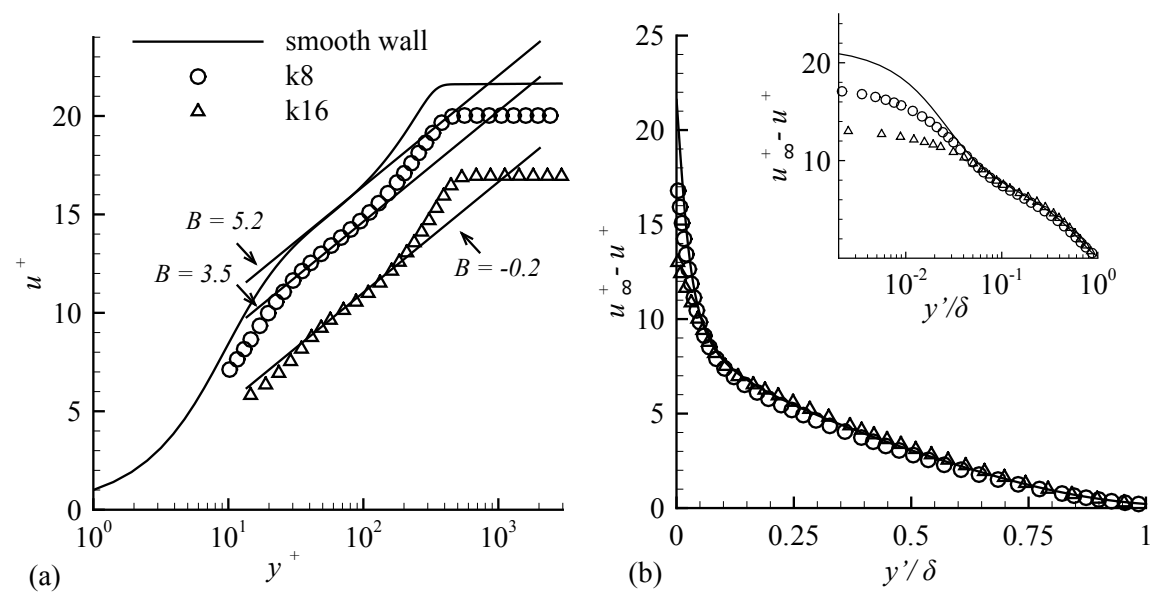

Fig. 16: Effect of increasing roughness height on the (a) mean velocity profiles and (b) defect profile. Inset shows the defect profile on a semi-log plot

cases. This corresponds to an axial location of $x / \theta_{0}=7000$ for the case $k 8$ and at $x / \theta_{0}=5000$ for case $k 16$. At these stations, $R e_{\theta}, u_{\tau}, k^{+}\left(=k u_{\tau} / \nu\right)$ for the cases $k 8$ and $k 16$ are around 1000, 0.05, 20 and 1200, 0.059, 47.2, respectively. Comparisons are made against the smooth wall case extracted at $R e_{\theta}=1000$ with a friction velocity of 0.046 . The effect of increasing roughness is manifested as an increase in the downward shift of the logarithmic region. The shift in the log-law can be quantified in terms of the roughness function, $\Delta U^{+}$which is around 1.7 for $k 8$ and 5.4 for $k 16$.

Figure 16(b) also presents the mean velocity profiles in velocity-defect form scaled in outer units. It is evident that the smooth and rough wall profiles collapse in the log-law and the outer region of the boundary layer. The inset shows the defect profiles on a semi-log axis demonstrating that the effects of roughness are confined within the overlap region. The trends are inline with the experimental observations of Schultz and Flack [41] and also support the wall-similarity hypothesis of Townsend [46].

Figure 17 compares the Reynolds stresses normalized by $u_{\tau}^{2}$ for the smooth and rough walls. The collapse of the Reynolds stresses in the outer layer is not as encouraging as that of the defect velocity profile. This trend is consistent with the observations of Lee and Sung [22]. They noted that the effect of roughness is observable in the outer layer. Deviation from the smooth wall profiles is much more predominant for the case $k 16$ when compared to $k 8$. This is attributed to the variation of $\delta / k$ in the similarity region $\left(x / \theta_{0}>5000\right)$. For the test case $k 8, \delta / k$ increases from $16.5-25$ while it is around $10-17$ for the $k 16$ case. The values are well under the threshold value of 40 proposed by Jiménez [20] for wall similarity. The outer flow is hence affected in the current study, due to an insufficient scale separation between the roughness height and the boundary layer thickness. 

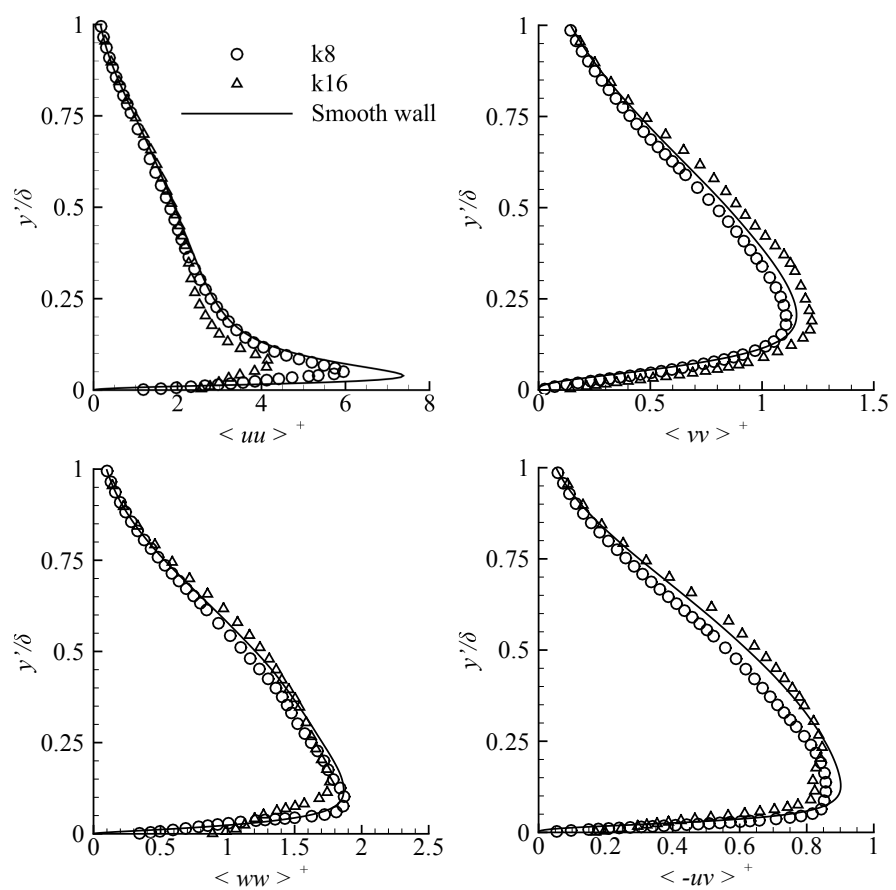

Fig. 17: Effect of increasing roughness height on the turbulent Reynolds stresses, normalized by $u_{\tau}^{2}$, in outer coordinates.

\section{Conclusions}

Transition of a subsonic boundary layer on a flat plate in the presence of roughness elements, distributed over the entire surface, is numerically investigated using a series of eddy resolving simulations. The effect of roughness on both the transitional and the subsequent turbulent boundary layers is explored. In the transitional regime, the onset of the flow transition predicted by the current simulations is in agreement with the correlations proposed by Stripf et. al. [45] and Redford et. al. [36]. The underlying transition mechanisms are shown to change significantly with an increasing roughness height. Roughness elements that are inside the boundary layer create an elevated shear layer. Alternating high and low speed streaks are observed underneath the shear layer. Secondary sinuous instabilities on these streaks destabilize the shear layer promoting transition to turbulence. For the roughness topology considered, it is observed that the instability wavelengths are governed by the streamwise and spanwise spacing between the roughness elements. In contrast, the roughness elements that are higher than the boundary layer create turbulent wakes in their lee. The scale of instability is much shorter and transition occurs due to the shedding from the obstacles. 
Post-transition, in the spatially developing TBL, the skin friction drag decreased and pressure drag increased with increasing surface roughness. For both the test cases $k 8$ and $k 16$, the roughness sublayer is confined within $4 k-$ $6 k$. The velocity defect profiles for both the smooth and rough walls collapsed when non dimensionalized in the outer units. However, when compared to the smooth wall, deviation in the Reynolds stresses are observable in the outer layer; the deviation being higher for the larger roughness elements.

\section{Compliance with Ethical Standards:}

Funding:

Author Nagabhushana Rao Vadlamani gratefully acknowledge the financial support from the St. Catharine's college, Cambridge through the Bowring research fellowship. The simulations are performed on UK Supercomputer ARCHER, to which access was provided through the UK Turbulence Consortium Grant No. EP/L000261/1. Computational time from Hartree centre (STFC) under Xeon Phi Access Programme is also acknowledged.

Conflict of Interests:

The authors declare that they have no conflict of interest.

\section{References}

1. Andersson, P., Brandt, L., Bottaro, A., Henningson, D.S.: On the breakdown of boundary layer streaks. Journal of Fluid Mechanics 428, 29-60 (2001)

2. Bernardini, M., Pirozzoli, S., Orlandi, P.: Compressibility effects on roughness-induced boundary layer transition. International Journal of Heat and Fluid Flow 35, 45-51 (2012)

3. Bons, J.P.: A review of surface roughness effects in gas turbines. Journal of turbomachinery 132(2), 021,004 (2010)

4. Bose, R., Durbin, P.A.: Helical modes in boundary layer transition. Physical Review Fluids 1(7), 073,602 (2016)

5. Brandt, L., Schlatter, P., Henningson, D.S.: Transition in boundary layers subject to free-stream turbulence. Journal of Fluid Mechanics 517, 167 198 (2004)

6. Braslow, A.L.: Review of the effect of distributed surface roughness on boundary-layer transition. Tech. rep., DTIC Document (1960)

7. Cardillo, J., Chen, Y., Araya, G., Newman, J., Jansen, K., Castillo, L.: Dns of a turbulent boundary layer with surface roughness. Journal of Fluid Mechanics 729, 603-637 (2013) 
8. Chatzikyriakou, D., Buongiorno, J., Caviezel, D., Lakehal, D.: Dns and les of turbulent flow in a closed channel featuring a pattern of hemispherical roughness elements. International Journal of Heat and Fluid Flow 53, 29-43 (2015)

9. Choudhari, M., Fischer, P.: Roughness induced transient growth. In: 35th AIAA Fluid Dynamics Conference and Exhibit, p. 4756 (2005)

10. Chung, D., Chan, L., MacDonald, M., Hutchins, N., Ooi, A.: A fast direct numerical simulation method for characterising hydraulic roughness. Journal of Fluid Mechanics 773, 418-431 (2015)

11. Citro, V., Giannetti, F., Luchini, P., Auteri, F.: Global stability and sensitivity analysis of boundary-layer flows past a hemispherical roughness element. Physics of Fluids 27(8), 084,110 (2015)

12. De Tullio, N., Paredes, P., Sandham, N., Theofilis, V.: Laminar-turbulent transition induced by a discrete roughness element in a supersonic boundary layer. Journal of Fluid Mechanics 735, 613-646 (2013)

13. Denissen, N.A., White, E.B.: Secondary instability of roughness-induced transient growth. Physics of Fluids 25(11), 114,108 (2013)

14. Ergin, F.G., White, E.B.: Unsteady and transitional flows behind roughness elements. AIAA Journal 44(11), 2504-2514 (2006)

15. Gaitonde, D.V., Visbal, M.R.: High-order schemes for navier-stokes equations: algorithm and implementation into fdl3di. Tech. rep., DTIC Document (1998)

16. Hack, M., Zaki, T.: Streak instabilities in boundary layers beneath freestream turbulence. Journal of Fluid Mechanics 741, 280-315 (2014)

17. Hong, J., Katz, J., Schultz, M.P.: Near-wall turbulence statistics and flow structures over three-dimensional roughness in a turbulent channel flow. Journal of Fluid Mechanics 667, 1-37 (2011)

18. Iyer, P., Muppidi, S., Mahesh, K.: Roughness-induced transition in high speed flows. In: 49th AIAA Aerospace Sciences Meeting including the New Horizons Forum and Aerospace Exposition, p. 566 (2011)

19. Jackson, P.: On the displacement height in the logarithmic velocity profile. Journal of Fluid Mechanics 111, 15-25 (1981)

20. Jimenez, J.: Turbulent flows over rough walls. Annu. Rev. Fluid Mech. 36, 173-196 (2004)

21. Lee, J.H., Sung, H.J., Krogstad, P.A.: Direct numerical simulation of the turbulent boundary layer over a cube-roughened wall. Journal of Fluid Mechanics 669, 397-431 (2011)

22. Lee, S.H., Sung, H.J.: Direct numerical simulation of the turbulent boundary layer over a rod-roughened wall. Journal of Fluid Mechanics 584, 125-146 (2007)

23. Leonardi, S., Orlandi, P., Antonia, R.A.: Properties of d-and k-type roughness in a turbulent channel flow. Physics of Fluids 19(12), 125,101 (2007)

24. Leonardi, S., Orlandi, P., Smalley, R., Djenidi, L., Antonia, R.: Direct numerical simulations of turbulent channel flow with transverse square bars on one wall. Journal of Fluid Mechanics 491, 229-238 (2003) 
25. Licari, A., Christensen, K.: Modeling cumulative surface damage and assessing its impact on wall turbulence. AIAA Journal 49(10), 2305-2320 (2011)

26. Loiseau, J.C., Robinet, J.C., Cherubini, S., Leriche, E.: Investigation of the roughness-induced transition: global stability analyses and direct numerical simulations. Journal of Fluid Mechanics 760, 175-211 (2014)

27. Matsuura, K., Kato, C.: Large-eddy simulation of compressible transitional flows in a low-pressure turbine cascade. AIAA Journal 45(2), 442457 (2007)

28. Montomoli, F., Hodson, H., Haselbach, F.: Effect of roughness and unsteadiness on the performance of a new low pressure turbine blade at low reynolds numbers. Journal of Turbomachinery 132(3), 031,018 (2010)

29. Muppidi, S., Mahesh, K.: Direct numerical simulations of roughnessinduced transition in supersonic boundary layers. Journal of Fluid Mechanics 693, 28-56 (2012)

30. Nikuradse, J.: Laws of flow in rough pipes. In: VDI Forschungsheft. Citeseer (1933)

31. Peet, Y., Sagaut, P., Charron, Y.: Pressure loss reduction in hydrogen pipelines by surface restructuring. international journal of hydrogen energy 34(21), 8964-8973 (2009)

32. Rao, V.N., Jefferson-Loveday, R., Tucker, P.G., Lardeau, S.: Large eddy simulations in turbines: influence of roughness and free-stream turbulence. Flow, turbulence and combustion 92(1-2), 543-561 (2014)

33. Rao, V.N., Tucker, P., Jefferson-Loveday, R., Coull, J.: Investigation of wake induced transition in low-pressure turbines using large eddy simulation. In: ASME Turbo Expo 2013: Turbine Technical Conference and Exposition, pp. V06CT42A008-V06CT42A008. American Society of Mechanical Engineers (2013)

34. Rao, V.N., Tucker, P.G.: Intake lip separation control using plasma actuators. In: 16th International Symposium on Transport Phenomena and Dynamics of Rotating Machinery (2016)

35. Reda, D.C.: Review and synthesis of roughness-dominated transition correlations for reentry applications. Journal of spacecraft and rockets $\mathbf{3 9}(2)$, 161-167 (2002)

36. Redford, J.A., Sandham, N.D., Roberts, G.T.: Compressibility effects on boundary-layer transition induced by an isolated roughness element. AIAA Journal 48(12), 2818-2830 (2010)

37. Reshotko, E.: Transient growth: A factor in bypass transition. Physics of Fluids 13(5), 1067-1075 (2001)

38. Rizzetta, D.P., Visbal, M.R.: Direct numerical simulations of flow past an array of distributed roughness elements. AIAA Journal 45(8), 1967-1976 (2007)

39. Roberts, S., Yaras, M.: Boundary-layer transition affected by surface roughness and free-stream turbulence. Journal of fluids engineering $\mathbf{1 2 7}(3), 449-457(2005)$ 
40. Schlatter, P., Örlü, R.: Assessment of direct numerical simulation data of turbulent boundary layers. Journal of Fluid Mechanics 659, 116 (2010)

41. Schultz, M., Flack, K.: The rough-wall turbulent boundary layer from the hydraulically smooth to the fully rough regime. Journal of Fluid Mechanics 580, 381-405 (2007)

42. Sengupta, A., Vadlamani, N.R., Tucker, P.G.: Roughness induced transition in low pressure turbines. In: 55th AIAA Aerospace Sciences Meeting, p. 0303 (2017)

43. Simens, M.P., Gungor, A.G.: The effect of surface roughness on laminar separated boundary layers. Journal of Turbomachinery 136(3), 031,014 (2014)

44. Smalley, R., Leonardi, S., Antonia, R., Djenidi, L., Orlandi, P.: Reynolds stress anisotropy of turbulent rough wall layers. Experiments in Fluids 33(1), 31-37 (2002)

45. Stripf, M., Schulz, A., Bauer, H.J., Wittig, S.: Extended models for transitional rough wall boundary layers with heat transferpart i: Model formulations. Journal of Turbomachinery 131(3), 031,016 (2009)

46. Townsend, A.A.: The structure of turbulent shear flow. Cambridge university press (1980)

47. Vadlamani, N.R.: Numerical investigation of separated flows in low pressure turbines. Ph.D. thesis, University of Cambridge (2014)

48. Vaughan, N.J., Zaki, T.A.: Stability of zero-pressure-gradient boundary layer distorted by unsteady klebanoff streaks. Journal of Fluid Mechanics 681, 116-153 (2011)

49. Visbal, M.R., Gaitonde, D.V.: Very high-order spatially implicit schemes for computational acoustics on curvilinear meshes. Journal of Computational Acoustics 9(04), 1259-1286 (2001)

50. Visbal, M.R., Gaitonde, D.V.: On the use of higher-order finite-difference schemes on curvilinear and deforming meshes. Journal of Computational Physics 181(1), 155-185 (2002)

51. Yuan, J., Piomelli, U.: Estimation and prediction of the roughness function on realistic surfaces. Journal of Turbulence 15(6), 350-365 (2014) 